Voix et Images

\title{
De la poésie et de quelques circonstances. Entretien avec Jacques Brault
}

\section{Robert Melançon}

Volume 12, numéro 2 (35), hiver 1987

Jacques Brault

URI : https://id.erudit.org/iderudit/200626ar

DOI : https://doi.org/10.7202/200626ar

Aller au sommaire du numéro

Éditeur(s)

Université du Québec à Montréal

ISSN

0318-9201 (imprimé)

1705-933X (numérique)

Découvrir la revue

Citer ce document

Melançon, R. (1987). De la poésie et de quelques circonstances. Entretien avec Jacques Brault. Voix et Images, 12(2), 188-211. https://doi.org/10.7202/200626ar d'utilisation que vous pouvez consulter en ligne.

https://apropos.erudit.org/fr/usagers/politique-dutilisation/ 


\title{
De la poésie et de quelques circonstances Entretien avec Jacques Brault
}

\author{
par Robert Melançon, Université de Montréal
}

\begin{abstract}
Si Boswell avait disposé d'un magnétophone, nous n'aurions pas The L ife of Johnson: tout gain technique entraîne une perte. Aussi, dès le début. Jacques Brault et moi avons-nous décidé d'en prendre à notre aise avec les transcriptions. Nous nous sommes rencontrés six ou sept fois de part et d'autre d'un magnétophone. d'avril à juin 1986. ce qui a produit une dizaine de cassettes. soit deux cents pages environ de texte brut. Nous avons coupć. déplacé. transposé, ajouté. Puis nous avons tout récrit, en respectant le caractère parlé de ces conversations.
\end{abstract}

Nous remercions Marie-Josée Sheitoyan, qui s'est chargée de la première et difficile transcription d'une partie des enregistrements, ct Johanne Lorion. qui a dactylographié un manuscrit surchargé.

R.M.

V. \& I. - Comment définiriez-vous la poésie?

J. Brault - II y a une saveur de l'existence, parfois très amère, qu'on peut éprouver indépendamment de ce qu'on fait ensuite. en insistant sur le mot faire, qu'on produise ou non des objets de langage. Je crois à cette expérience non pas simplement en vertu d'un présupposé ou par un a priori. J'ai rencontré quelquefois des êtres qui n'avaient rien à voir avec la littérature, la philosophie, les arts. À mes yeux, ils étaient sensibles à cette poésie de l'existence qui tient simplement à la capacité daccueillir les concentrés vitaux dans la vie quotidienne: tout à coup, il y a cristallisation de l'instant. Ça peut être tout à fait heureux, malheureux, ou encore de l'ordre du grand comique ou du tragique. Ainsi, jaai rencontré quelqu'un - ça peut sembler étrange: il était notaire de son métier et les notaires ont mauvaise réputation quand on parle de poésie... J'étais adolescent, et j’étais allé passer une semaine à l'Abbayé de Saint-Benoît-du-Lac. Ce notaire était là et nous avons pu nous connaître un peu. C'était un homme d'une soixantaine d'années. Je ne sais s'il était en veine de confidences: peut-être avait-il gardé le silence longtemps avant que j'arrive... Il me parlait très librement, à bâtons rompus, et j’ai été frappé de voir qu'il arrivait dans une conversation quotidienne à décanter dans ce qui semblait avoir été sa vie, la grâce de l'instant: il y a comme ça quelque chose tout à coup, qui nous est offert. Moi, j'y crois, un peu bêtement, je l'avoue, et je serais bien en peine de le prouver. 


\section{V. \& 1. -- Comment cette grâce se manifeste-t-elle?}

J. Brault - Il y a une capacité d'accueil par quoi l’on reconnait ces événements, mais on ne les reconnait pas nécessairement sur le plan intellectuel, on ne les analyse pas. Ce notaire me parlait, mais c'était moi qui faisais un tri et qui accollais des valeurs plus ou moins poétiques à ses propos. Lui, il me racontait des moments de sa vie, il me décrivait sans y penser sa façon d'être au monde. À l'époque, j’avais une perception globale et un peu confuse. J'en parle maintenant avec du recul, après m'être livré à une analyse, au sens large du mot, qui m’a fait conclure à ce que j'ai dit tout à l'heure. Comment ce sentiment de poésie se manifeste-t-il? Il doit y avoir des variables selon les tempéraments, les cultures, les époques, les lieux, les individus. Il y a au moins une constante. que je définirais par le mot donné ou par celui de grâce. Ça demeure un peu mystérieux pour moi, mais je continue à y croire: un instant parmi les instants, à cause des dispositions dans lesquelles on se trouve ou de la conjonction d'une foule de facteurs, prend un air d'éternité. II y a ce que Stendhal appelle cristallisation... Ce n'est pas, d'entrée de jeu, littéraire. Ça peut être vécu dans le silence. et ça n'est pas nécessairement porteur de possibilités langagières. Comme toutes les sensations un peu profondes qu'on peut avoir dans une vie. Ça peut être un timbre de voix... Je vais le raconter même si ça peut avoir l'air assez ridicule : à l'université, il y a des années, j'entendais un certain pas dans un couloir. Je l'ai entendu plusieurs fois - ma porte de bureau était entrouverte:-je me sentais un peu prisonnier de ma cage - et ce pas-là, dont je n'ai pas oublié le rythme et, si je peux dire, le timbre. m'a fait énormément de bien, m’a rejoint je ne sais dans quelles zones d'être. C'est un exemple de ce que j'appelle une manifestation d'ordre poétique.

V. \& 1. - Cette poésie qui n'est pas organisée par le langage, n'est-ce pas la mémoire qui va la former à partir des accidents de l'existence? Ce pas dans le couloir, agissait-il à l'instant même ou plus tard, dans le souvenir que vous en gardiez?

J. Brault - L'un et l'autre. Au moment même, quelque chose s'est produit. Bien sûr, on parle toujours au passé finalement, on ne parle jamais de façon contemporaine ou concomitante. Et la mémoire va tout passer au crible par la suite. Mais je crois très fortement à ce que j'appellerais le moment indépassable non seulement de la perception mais de la sensation, qui n'est même pas la reconnaissance d'un sens. qui peut demeurer énigmatique et garder quelque chose du caractère brut des sensations primaires de froid ou de peur. Je suis obligé de dire que j’y crois parce que j'ai de la difficulté à le démontrer, mais je pense qu'à l'origine de toute poésie. même de la plus quintessenciée doit se trouver cette expérience primordiale. Ce n'est pas toujours quelque chose qui nous met en contact avec une personne ou avec un objet identifiables; ça peut être un état d'être, une façon d'être. Autrement dit, il y a une poésie de la relation de l'humain et du monde. Mais effectivement, le souvenir, la mémoire vont venir interférer par la suite: «je me souviens qu'il y a eu telle chose"... Bien. Mais entre ce (je me souviens» et telle chose, il y a un espace, un temps, qui ont servi à réaménager la sensation. 
V. \& I. - Peut-on dire que cette expérience a lieu au moment où le contact avec le monde se fait énigme?

J. Brault - Oui, et pour cette raison il est difficile d'en parler ou de l'analyser: c'est une énigme. On a prise sur ses manifestations: des formes, des matières. des choses qui nous ont frappé. qui vont rester dans la mémoire, qui vont être véhiculées à travers tous les aléas et les vicissitudes de l'existence. Mais ça demeure une énigme, ce n'est pas clair, même si c'est exaltant. Ça demeure un secret. Paulhan disait, je ne me souviens plus où et je cite de mémoire, sans doute très approximativement, que le secret des cuvres littéraires qui valent. c'est que. justement. elles ont un secret. Ce n'est pas par une analyse. par un examen qui se voudrait exhaustif qu'on arrivera à épuiser ce secret, à le faire se révéler ou à percer son énigme. Au départ. l'essentiel est là. Ensuite. la manifestation par différents moyens artistiques n'est pas une explicitation du secret. On danse autour, on s'approche peu à peu, on s'aperçoit qu'en s'approchant on s'est peut-être éloigné: tous les paradoxes se donnent cours. Finalement, on signale, on laisse se signaler la présence de l'énigme ou du secret dans ce qu'on appellera par la suite le poème.

\section{V. \& I. - On cherche à préserver l'énigme?}

J. Brault - Souvent, oui. Jai horreur des poèmes qui sont explicatifs, consciemment ou non, parce qu'en général ils sont plats. Peut-être vient-on à la poésie justement pour préserver l’énigme, non seulement pour la conserver mais pour la mettre à l'abri parce qu'elle contient la plus précieuse valeur de l'existence.

V. \& I. - Comment passe-t-on de cette expérience au poème? Tout le monde peut éprouver ce mystère de l'existence, mais tout le monde ne se met pas à écrire.

J. Brault - Le langage vient croiser cette sensation muette. Cé peut être du langage embryonnaire comme chez certains poètes japonais: «je vois... ça... ça...». Ou encore le simple fait de reconnaître qu'on est sans paroles, qu'on n'arrive pas à dire. $\grave{A}$ mes yeux. paradoxalement, c'est du langage: même lorsqu'on se demande intérieurement de façon confuse: "qu'est-ce que c'est que ça?", en fait, c'est déjà du langage. Je ne crois pas au langage vide ou qui se nourrit de lui-même, "rendre plus purs les mots de la tribu» et le reste: ça m'alerte toujours... Je crois que la poésie de langage doit coïncider avec l'expérience, s'accrocher à elle; il faut que cette énigme se transpose dans un matériau gros en lui-même de possibilités. Des mots nous viennent, une séquence, des échos - certains parlent d'un rythme, c'est possible mais ça me semble un peu abstrait... Je me souviens, pour donner un exemple, qu'un poème m'était venu quand je surveillais un examen. Il y a de cela très longtemps. J'étais professeur dans un collège, c'était la fin de l'année, le début de l'été donc. Il faisait beau, les fenêtres étaient ouvertes... Je m'ennuyais et je rêvassais. Que s'est-il passé? Je ne le sais pas. Chose certaine, quelque chose m’avait frappé quelque temps auparavant - une semaine, un mois, un an plus tôt, peu importe. Quelques mots me sont venus. J'étais fatigué de lire; 
puis, il est toujours ennuyeux de surveiller un examen... J'ai pris une feuille... Ce n'était pas de l'automatisme, pas du tout. J'ai écrit et j'ai cru reconnaître une chose qui était arrivée, qui n’avait pas de nom, à travers ces mots qui venaient... Au bout d'une demi-heure, j'avais écrit un petit poème, d'une traite; ça ne m'est pas arrivé très souvent. Par la suite, je l'ai corrigé, je l’ai retravaillé mais j’ai changé peu de choses. Ce qui avait l'air au début d'être un magma verbal s'est révélé la fin de mon texte. Ce n'était pas de l'association pure et simple; il y avait un certain déroulement, une certaine organisation... Ce n'est qu'ensuite que je me suis posé des questions et que j'ai reconnu la présence de quelque chose que j’avais éprouvé obscurément -quand? où exactement? ça restait vague. Quelque chose m'échappait en partie. Au fond, on ne sait pas très bien, non seulement ce qu'on veut dire mais ce qu'on dit, même si, par ailleurs, la connaissance artisanale, comme dans toute manipulation d'un matériau, nous permet de dire que ceci va. que cela ne.va pas, ou. que ce n'est pas au point, qu'il faudrait le refaire. Mais fondamentalement, il nous faut reconnaître si ce à quoi on a affaire vaut la peine d’être travaillé ou si, non. il n'y a pas eu cela, qui est le déclencheur.

V. \& I. - Mais pourquoi des mots? Chez d'autres, cela pourra donner lieu à un dessin, à une musique...

J. Brault - Je ne le sais pas. J'avoue que ça m'échappe. Tout le monde entend, voit, est doué de langage...

V. \& I. - Avez-vous déjà songé à peindre ou à composer de la musique au lieu d'écrire?

J. Brault - Non. Pour moi, dès le début, c'était une question de langage. En musique, malheureusement, j'ai été et je reste un peu sourd; je n'ai pas reçu l'éducation qu'il aurait fallu, et j’ai le préjugé que l'accès à la musique doit être précoce. Les couleurs, les formes m'intéressaient. mais j'étais davantage sollicité par le langage, par les mots, avec d'ailleurs une conscience douloureuse - mais il s'agit peut-être de souvenirs reconstitués dont il faut se méfier - d'être très pauvre en ce domaine. Je serai très clair, donc injuste: j'ai senti, très jeune, vers dix ou douze ans, que je n'avais pas beaucoup de langage. La lecture me passionnait, je savais un peu ce que c'était que le langage. J'avais un frère aîné qui avait le droit d'aller à la bibliothèque; moi, j'étais trop petit; alors il m'empruntait des livres - je lui passais mes commandes - parce que c'était moi le liseur. Il m'arrivait de préférer lire à jouer, ce qui marque une attirance certaine: pour qu'un enfant renonce à une bonne partie de ballon pour lire, non pas parce qu'il est fâché avec'ses camarades ni malade, il faut un puissant motif. J'étais sportif, et j'aimais jouer; je renonçais donc à ma partie de ballon pour lire, mais c'était d'autant plus délicieux - parfois c'était même un dilemme. C'étaient des lectures d'enfant. C'était vraiment la magie des mots parce qu'il n'y avait pas alors ces albums illustrés qui sont si attirants aujourd'hui. Même ma première grammaire, je l'ai lue et relue; elle contenait des exemples tirés des meilleurs auteurs de la langue française, dont La Fontaine... Pour moi, c'était une lecture. Indéniablement j'aimais les mots. Mais pourquoi? comment? je ne le sais pas. 
V. \& I. - Il y a eu un saut dans l'écriture que tous les liseurs ne font pas.

J. Brault - Je pense que la différence - je ne veux pas proposer de théorie, je parle d'expérience - s'explique par ce fait que le liseur qui est attentif à la façon dont ce qu'il lit est fait se trouve au bord de l'écriture. Il ne va peut-être pas nécessairement écrire. Mais en même temps, sans que son plaisir en soit gâché, il s'est avancé dans la direction de l'écriture, plus que celui qui accueille simplement le résultat. Ce sont des choses qu'on connaît peu. Peut-être y $a-t-i l$ chez certains un désir plus poussé, d'imiter. Je crois que tout commence par l'imitation, la reproduction même. À quoi cela tient-il? Qu'est-ce que ce désir vient éveiller? De quelles profondeurs sort-il? J'avoue ne pas avoir de réponse. Très tôt je me faisais des cahiers où je notais des phrases que je trouvais belles, en tant que phrases, une sorte d'anthologie personnelle. Et j'aimais lire dans le dictionnaire. Mais je sais bien que tout cela ne permet pas de trancher la question: certains lecteurs vont aimer fouiller dans des grammaires, même noter de belles phrases, sans jamais écrire eux-mêmes. Quel est le passage, je ne le sais pas.

V. \& I. - Vous avez évoqué la conscience du manque de mots. Pourrait-on dire que celui qui n’éprouve pas de difficultés avec le langage n’écrira pas?

J. Brault - Je serais tout à fait d'accord. Je crois qu'on devient écrivain pour guérir, ou plutôt pour adoucir une angoisse, une angoisse verbale qui n'est pas uniquement extérieure, qui est une façon d'être mal dans le langage... Certaines angoisses sont actives, comme d'autres peuvent être paralysantes. tout comme certaines maladies peuvent être créatrices. Certains créateurs ont été longtemps malades et sur leur maladie même prélevaient des pulsions créatrices. Je dis "actives" parce que pour créer il faut travailler. Peut-être cette angoisse spécifique entraîne-t-elle une rumination et une descente dans l'abîme du langage. Quelqu'un qui serait comblé de ce point de vue fera un causeur...

V. \& I. - Pourtant chacun connaît des causeurs qui s’expriment de façon abominable, très imprécise, mais qui sont manifestement très heureux de parler et qui prennent la parole indéfiniment, sans quartier. Alors que d'autres, qui maîtrisent le langage, vont éprouver plus de réticences à s'exprimer. Pourrait-on dire que le langage se met à faire problème parce qu'on est écrivain?

J. Brault - Cette difficulté n'est peut-être pas une cause mais une circonstance, une occasion. Même un écrivain qui court tous les risques qu'il faut acquiert du métier; on pourrait donc croire qu'il en arrivera à se donner des moyens. Mais l'histoire littéraire nous enseigne toutes sortes de choses contradictoires : certains donnent leur œuvre au début. même si les Rimbaud ne courent pas les rues; d'autres atteignent à une espèce d'acmé; d'autres sont plus tardifs; d'autres ont des hauts et des bas; d'autres se maintiennent. Tant de choses entrent en considération qu'il est très difficile de donner des règles générales. 
V. \& I. - À mesure qu'un écrivain acquiert de la maîtrise, peut-être rencontre-t-il de nouvelles exigences plus considérables que la maitrise qu'il vient d'acquérir.

J. Brault - Oui. En même temps, s'il n'est pas complètement idiot ni trop enfermé en lui-même, il doit bien mesurer cette terrible distance, se dire: «non, je n’y suis pas encore», tout en acquérant d'autres possibilités.

V. \& I. - Quand ce passage de la lecture à l'écriture s'est-il effectué dans votre cas? Depuis cette anthologie de belles citations...

J. Brault - Certaines lectures font date: celle de Baudelaire. II n'était pas permis, en tout cas pas très conseillé de le lire quand j'étais au collège. J'avais quinze ans; je suis allé à la bibliothèque municipale. Je revois encore les Fleurs du mal dans l'édition Variétés - elle n'était pas très bonne mais peu importe. J'ai eu de la difficulté à m'en remettre: ça cognait dur. J'avais lu un peu de Verlaine avant; je n'avais sans doute pas saisi toutes les subtilités, je lisais à l'aventure... Baudelaire a marqué une étape. À seize ans, tout de suite après, Rimbaud. Puis, à dix-sept ans, Mallarmé : j’ai grincé des dents mais, quand même, ne serait-ce que comme repoussoir... Il y a des lectures déterminantes. Pourquoi celles-là? Ce peut être simplement parce que Baudelaire m'est tombé sous la main, ou qu'il m'intriguait parce que j'en avais entendu dire des choses étranges et contradictoires; Rimbaud, ça se comprend: il était collégien comme moi et j'étais drôlement curieux d'y aller voir. En même temps. j’ai lu Saint-Denys Garneau, qui était tout près, comme quelqu'un de la paroisse. Ces lectures changent les perspectives, même si. moi, j’ai été très long à me mettre à écrire. Non. Je devrais faire une distinction: j'écrivais beaucoup; au collège on nous faisait écrire. En classe de belleslettres, un professeur nous a fait acheter deux forts cahiers; nous pouvions arriver librement une demi-heure avant le début des cours, le matin, pour écrire sur un thème donné, le plus souvent une citation de grand écrivain transcrite au tableau. J'ai écrit toute l'année (on avait deux cahiers pour que l'un soit corrigé pendant qu'on écrivait dans l'autre) et j’ai découvert ainsi des auteurs qui n'étaient pas au programme, par exemple Joubert. Ce professeur corrigeait intelligemment, sans la moindre censure. uniquement la langue, sans nous gronder sur nos opinions; c'était gratuit, indépendant de toute note. Puis je lisais Baudelaire et Rimbaud; alors j'ai écrit des masses d'alexandrins et de décasyllabes, des sonnets, forme que je trouvais très difficile et très contraignante. C'était écrire, en un sens. Ça peut sembler un peu ridicule ou gratuit à distance, mais pas du tout. Par contre, avoir conscience et volonté d'écrire, comme un jeune écrivain, ou même, éventuellement, publier des choses, c'est venu plus tard. Au.début de la vingtaine, je me suis senti bien démuni, pauvre à tous les points de vue, pauvre de moyens et même d'objectifs. J'ai publié de petites choses ici et là, mais en ayant conscience que j'étais en train d'apprendre un métier. Ce n'est qu'à la trentaine que tout s'est déclenché. Il m'a fallu quinze ans pour consentir à être. peut-être, un écrivain, je dis bien «consentir». 


\section{V. \& I. - Comment écrivez-vous maintenant?}

J. Brault - Selon les circonstances, qui jouent un rôle important, je le constate: beaucoup de choses que j’ai écrites m'ont été commandées, peutêtre pas toujours de façon très précise et je restais libre d'en disposer. Souvent je me suis cru paresseux mais j’avais peut-être besoin d'une incitation à écrire, un peu comme si je continuais à croire que je ne suis pas vraiment écrivain. J'ai écrit continuellement. mais j'ai publié très sporadiquement. J'ai toujours continué à écrire dans mes cahiers; je pense que ça m'est venu de ce que j'évoquais tout à l'heure, du collège. J'ai des cahiers... J'en ai jeté beaucoup. Ce sont des fourre-tout, qui n'ont rien à voir avec le genre journal: je n'ai jamais été capable d'en tenir, c'est aux antipodes de tout ce que je suis. Par contre, prendre des notes, accumuler du matériel, griffonner, barbouiller. je le fais continuellement. C'est comme ça que je travaille, souvent au gré des sollicitations que j'utilise comme «déclencheur», et pour. le reste. de façon très libre. Je n'ai jamais voulu devenir écrivain professionnel, parce qu'un professionnel. par définition, va vivre de ce qu'il écrit, parce qu'il va y consacrer le plus clair de son temps et de ses énergies - ce qui ne m'aurait pas intéressé. Me sentir obligé de sortir un livre tous les trois ans, ça me rendrait malheureux ; être présent à gauche et à droite dans des publications, des revues. parce que ce n'est pas tout que d'arriver, il faut se maintenir... Non. De ce point de vue-là. je me sens parfaitement libre. Mais le sentiment du manque persiste.

V. \& I. - Qu'attendez-vous de la poésie? Qu'attendez-vous, en particulier, de celle que vous écrivez?

J. Brault - C'est une question difficile. Aujourd'hui, comme j'ai l'impression que la poésie me manque depuis quelques années, ou que je lui manque, je me pose de moins en moins de questions de cet ordre. Je n’ai jamais décidé d'écrire tel type de texte, par exemple du théâtre, ou du roman, ou des poèmes. Même pour des articles critiques qu'on me demande pour des revues universitaires, j'en prends à mon aise; s'ils ne sont pas contents, ça ne me vexe pas. Je ne me laisse plus téléguider. jamais. Cela dit, ce que j’attends de la poésie, aujourd'hui, c'est de vivre moins bêtement, de ne pas crever dans la stupeur. Cela semble négatif. mais c'est quand même quelque chose. J'attends de ne pas glisser tout à fait dans le non-sens. Au fond, j'attends peu de choses de la poésie. Enfin, peu de choses... Je n'en attends pas monts et merveilles: le "salut", la révélation de je ne sais trop quoi... Tout simplement. à travers l'amertume de la vie - je ne parle pas nécessairement de ma vie individuelle mais de la vie tout court - une saveur. J'aime le mot saveur: il inclut le sensible. Une certaine saveur qui n'est pas à dédaigner même si elle n'est qu'éphémère. C'est un peu cela que j'attends de la poésie. Une façon d'être. Quand j'en lis, j'en entends, j'en vois, quand ça m'arrive, quand ça m'est proposé, quand je peux être disponible, c'est quelque chose de cet ordre-là qui se produit. Quand moi-même j'essaie d'écrire ce qu'on appelle habituellement un poème, les meilleurs moments (ça n'est pas pendant, parce qu'alors c'est dur et souvent on ne sait pas où on va et c'est du travail) c'est avant, une espèce de pressentiment non pas tant qu'on va écrire un poème 
mais qu'il y a quelque chose; et, je l'avouerai, après, il m'est arrivé quelquefois, indépendamment de ce que ça valait, d'éprouver un bonheur très spécial, très particulier. Ensuite, ça s'estompe, bien sûr... Éprouver certains bonheurs qui, si fugitifs qu'ils puissent paraître maintenant, ne m'ont quand même pas complètement abandonné. Ça n'est déjà pas si mal...

\section{V. \& I. - Vous arrive-t-il de chercher la poésie?}

J. Brault - Oui, certainement. Je ne dirais pas de la chercher directement mais d'aménager des circonstances propices, le lieu. le temps, de m'aménager moi-même de façon très concrète, soit par la lecture, soit par la rêverie, par des promenades ou des ruminations, ou par le silence qu'il faut beaucoup cultiver. Ou simplement de faire des jeux verbaux. J'appelle ça des jeux, mais ce sont plus que des jeux: c'est un peu comme si je piquais doucement une bête endormie sans savoir si elle va me sauter dessus ou si, au contraire, elle va se montrer une compagne fidèle, ne serait-ce qu'un court espace de temps. Mais c'est surtout sa propre mise en état qui importe, et je dirais qu'elle demande beaucoup de silence, beaucoup d'oubli de soi, parce que s'il y a trop de conscience au départ... J'ai fait quelques expériences. Celle de la traduction, en particulier, m'a été bénéfique. Je ne suis pas traducteur de profession. Dieu merci; c'est un dur métier, et comme je suis un peu paresseux, je ne l'aurais pas choisi. Des expériences de traduction m'ont parfois préparé à la poésie, oui. Ou bien je note encore dans des cahiers des choses d’autres écrivains, un poème, un extrait; je les transcris à la main; il ne me viendrait pas à l'esprit de les mettre dans un ordinateur, ça ne serait pas la même chose. Le fait de les transcrire au stylo sur une belle page propre mamène souvent au bord de quelque chose. Je dis "au bord»... Puis il arrive qu'on écrive des choses qui nous semblent aller dans telle direction, devoir prendre à peu près telle forme, et qui changent de sens. Voilà.

V. \& I. - Vous semblez attendre la même chose de votre poésie que de celle des autres.

J. Brault - Oui, exactement, c'est la même chose en lecture et en écriture. Comme lecteur, je sais que j’ai dû sans doute passer à côté de choses importantes, que j'en ai peut-être laissé tomber avant le temps, mais peu importe. Quand même, quand on pense à tout ce qui est disponible... Mais il ne faut pas que ce soit une attente trop active. Je considère que quand j'ai fourni un effort honnête... Il y a des choses qui se sentent. J'ai l"habitude de renifler les textes: il faut qu'il se passe quelque chose à ce moment-là, qu'on sente cette énigme, ce noyau noir et infissible, sinon, bon, ça peut être intéressant pour le métier, pour toutes sortes d'autres raisons que la poésie. Ce que j'aime maintenant comme lecteur libre, c'est dialoguer, durement au besoin, durement pour moi. Cela peut inciter à l'écriture, et pas nécessairement à l'écriture critique.

V. \& I. - Quelles sont les œuvres qui vous sollicitent? Tantôt, vous avez nommé Baudelaire; vous avez préparé l'édition des Oeuvres de Saint-Denys Garneau... 
J. Brault - Je ne sais si c'est une règle pour tout le monde: j'ai souvent entendu des gens d'un certain âge dire qu'on en vient à relire de plus en plus. Je ne sais si c'est vrai. Moi, je continue à lire. Évidemment, je ne suis pas du genre à me mettre à l'affût de la production courante. J'aime beaucoup d'auteurs. Je ne suis pas très fétichiste, ni même admirateur; je suis plutôt un lecteur amoureux. parfois déçu mais peu importe. Ce qui m'intéresse. c'est d’établir toutes sortes de connexions entre mes lectures et mes auteurs. Même les plus incongrues: pourquoi pas entre Baudelaire et Rabelais? À première vue, ça semble absurde. mais ça ne l'est pas dans l'aménagement de mes lectures. Puis certaines choses se décantent: je vais relire quelques poèmes de Saint-Denys Garneau, quelques pages dans ce qu'on a appelé son Journal - ce sont en fait des cahiers -.. quelques lettres, non pas que je trouve le reste mauvais, mais il faut faire des choix. Dans les nouveautés, je vais à la pêche, parfois...

V. \& I. - Cette façon de lire laisse entrevoir une préférence pour un type de textes. Est-ce que je me trompe en suggérant que vous préférez. ceux qui sont écrits par fragments?

J. Brault - Oui, plutôt. Je crois même que comme écrivain, pour se lancer dans un livre de cinq ou six cents pages d'une pièce, il faut être un peu effronté, et que, comme lecteur, il faut être fermement décidé. Je n’ai rien contre les pavés, la question n’est pas là. Mais même dans des œuvres de longue haleine, par exemple Christine Lavransdatter de Sigrid Undset, j'ai prélevé des moments privilégiés à ma seconde lecture; maintenant, je peux en relire certaines parties avec un plaisir accru, mais je ne sens pas le besoin de le relire en entier. Avec mon très cher Stendhal, je procède de la même façon, même si j'ai relu quelquefois intégralement la Chartreuse de Parme pour laquelle j'ai un faible. Je me sens plus à l'aise dans Montaigne, où on peut butiner à son aise, établir toutes sortes de connexions. Quant à la poésie, elle se prête à cette lecture, par définition. parce qu'elle est plus mobile. Même en philosophie, je procède aussi par prélèvements, dans Kierkegaard, dans Nietzsche...

V. \& I. - Ce mode de lecture entraîne que vous ne fréquentiez pas certaines cuvres qui s'y prêtent mal.

J. Brault - Oui. Dans les œuvres anciennes, je suis beaucoup plus à l'aise chez Platon que chez. Aristote. Platon a une pensée aussi ferme et aussi organisée mais Aristote est systématique et didactique. tant et si bien qu'il est difficile de s'en tenir à tel ou tel chapitre parce qu'il est fonction de l'ensemble. Tandis que chez. Platon. il y a des moments de grâce qui ont leur autonomie. Même dans le Banquet, que je relis souvent, je saute le discours d'Agathon et d'autres, et je me recompose mon Banquet à moi. Je sais que c'est indéfendable aux yeux d'une certaine critique, mais c'est une position de lecteur.

V. \& I. - Dans un banquet, pourquoi un convive ne s'absenterait-il pas pendant un discours... 
J. Brault - ... qui l'intéresse moins ou qu'il a déjà entendu ou dont il croit qu'il n'a plus rien à tirer. Quand Alcibiade arrive, pour moi, il se passe toujours du nouveau et ce que j'appelle le poème de Diotima reste insondable. J'ai déjà lu tous les discours qui servent de préparatifs, mais maintenant ce n'est plus nécessaire. Je me suis fait mon grand recueil de fragments ou de morceaux. et c'est cela mon livre de lecture. C'est celui que j'apporterais sur une île déserte.

V. \& I. - Ce livre laisse entendre que, toutes époques et tous genres confondus, il y a quelque chose de commun à un fragment de Platon, à un poème de Baudelaire ou de Saint-Denys Garneau, à une note de Joubert. Quoi?

J. Brault - Le commun dénominateur? J'ai failli répondre spontanément la poésie, mais ce n'est pas seulement celle du poème formellement constitué. C'est une poésie bien particulière, parce que j'ai horreur de la prose poétique, comme d'ailleurs des films poétiques ou du théâtre poétique... Il y a tout un poétique qui sent le frelaté et qui appartient probablement à l'ordre des retombées de la poésie. C'est la poésie-alibi: on pourrait tout dire en ne disant rien. 11 y a toute une poésie qui n'est qu'une manière de ne pas écrire: enfiler des mots, bricoler des choses sans suite... Il peut se produire. à l'occasion. d'heureuses rencontres, mais ça ne m'intéresse pas de chercher deux ou trois pierres précieuses minuscules dans une masse informe. La poésie telle que je l'entends suppose la fermeté du langage, une certaine économie - j'ai horreur de sentir qu'il y a une page de trop dans un texte. C'est peut-être par analogie que je parle de poésie pour dire texte serré, le poème étant une forme plus courte parce qu'elle se veut plus dense, et plus ambitieuse. finalement, un peu plus autonome même si elle n'est pas fermée sur elle-même. Le poème comme je me le représente tend à une certaine auto-suffisance. Par analogie, c'est ce que j'aime retrouver en prose, parce que je lis la prose comme je lis la poésie, et non pas l'inverse. Je ne cherche pas les sentiments, les belles images. Dieu sait quoi; je cherche la justesse. pas nécessairement la justesse grammaticale mais celle de l'ensemble, des parties et des proportions, celle du propos - si le propos est égaré, je cherche la justesse de l'égarement et ainsi de suite. J'aime beaucoup certains écrivains dits secondaires comme Jacques Chardonne, pour prendre l'exemple d'un écrivain français du $X X X^{\mathfrak{C}}$ siècle. Je n'aime pas tout, mais une bonne partie de son cuvre. même si je trouve que c'est un penseur exécrable. Indépendamment de cela, je l'aime beaucoup. C'est la même chose pour Marcel Arland dont j'aime le style un peu plus parlé mais qui, justement, a de beaux raccourcis. Ils ne sont ni l'un ni l'autre très courus ni très célébrés. D'autres fois, à l'inverse, je trouve que chez Malraux, c'est du charrié, que chez Gide, cest de la haute grammaire, de la haute voltige et beaucoup d'autosatisfaction. Ce n'est pas un jugement moral: c'est comme si une phrase se mirait dans la suivante et se trouvait bien faite. J'aime par dessus tout ce qui est à la fóis abandonné et contrôlé.

V. \& I. - Vous préparez une réédition de vos premiers recueils: peut-on retoucher un texte écrit et publié il y a plusieurs années? 
J. Brault - On le peut dans une certaine mesure. Je reprends Mémoire, la Poésie ce matin et l'En dessous l'admirable. Dans Mémoire, je suis allé à la partie la plus ancienne, "Quotidiennes". je me suis dit: «jetons un regard critique" et j'ai sorti mon scalpel: J'ai retouché un petit poème qui avait d'ailleurs été exclu de la version publiée chez Grasset et que j'ai réintroduit; j'en ai modifié un autre, et c'est tout. avec quelques légères retouches ici et là. très peu. Je me suis arrêté. parce que c'est derrière moi. non pas que ce soit si loin dans le temps mais c'est loin dans le mouvement. Il aurait fallu que je retrouve cette sensation dont je parle toujours et sur laquelle je ne m'explique jamais, ou que je fasse autre chose. Et quant à faire autre chose, autant le faire ailleurs. J'ai été incapable d'y revenir. Non que je pense qu'il n'y aurait rien à reprendre ou à retrancher, mais si je retranche ici, ce qui reste ailleurs sera déséquilibré. J'ai longuement hésité et j'ai pris, pour l'instant, la décision de ne pas revenir sur ces textes.

V. \& I. - Vous vous êtes donc lu vous-même attentivement, à distance de vingt ans. Je suppose qu'on ne fait pas cela souvent...

\section{J. Brault - Non.}

V. \& I. -- Comment vous êtes-vous lu?

J. Brault - D'abord je trouve un peu déprimant de me lire. Je ne le fais que si je suis obligć. Je vois les lacunes, les occasions ratées, tout un négatif qui me saute aux yeux. Jai essayé d'être sans préjugés, d'être honnête avec moi-même. Je voyais beaucoup de modifications à faire, mais je n'étais pas capable de les faire. Je me suis relu aussi avec un certain sentiment d'étrangeté ; j’ai éprouvé qu'une distance s'était creusée entre celui que je suis maintenant et ces textes. Je me sentais comme celui qui marche dans le désert. qui se retourne et qui voit ses traces s'effacer avant même d'atteindre la ligne de l'horizon. Je l'ai fait parce qu'il le fallait. mais je ne me retourne pas spontanément sur moi-même. J'ai éprouvé surtout un sentiment d’étrangeté. $\mathrm{Ce}$ n'est pas une question d'années: cela peut m'arriver avec des choses écrites il $\mathrm{y}$ a six mois.

V. \& I. - Cela force à poser la question énorme de l'identité. Y a-t-il quelque chose' qui dit «je» ou ne sommes-nous qu'une suite d'instances successives?

J. Brault - Je constate une certaine cohérence à travers tout ce qui varie. Il y a donc une certaine identité personnelle.

V. \& I. - Alors d'où vient ce sentiment d'étrangeté?

J. Brault - Je ne sais pas. Ça doit être congénital. Comment dire? Je m'intéresse si peu à moi-même. Je ne me suis pas posé cette question. Disons que je m'oublie volontiers en chemin. Pour moi, ce qui importe, c'est de cheminer. On m'a déjà dit que le chemin était une de mes marottes. C'est bien possible. Il y a des haltes, bien sûr. mais on ne pense à soi que très vaguement, dans une sorte de brouillard. Ce qui me mobilise plutôt, c'est. je ne sais pas, l'idée qu'il y a en moi le visage de l’autre. 


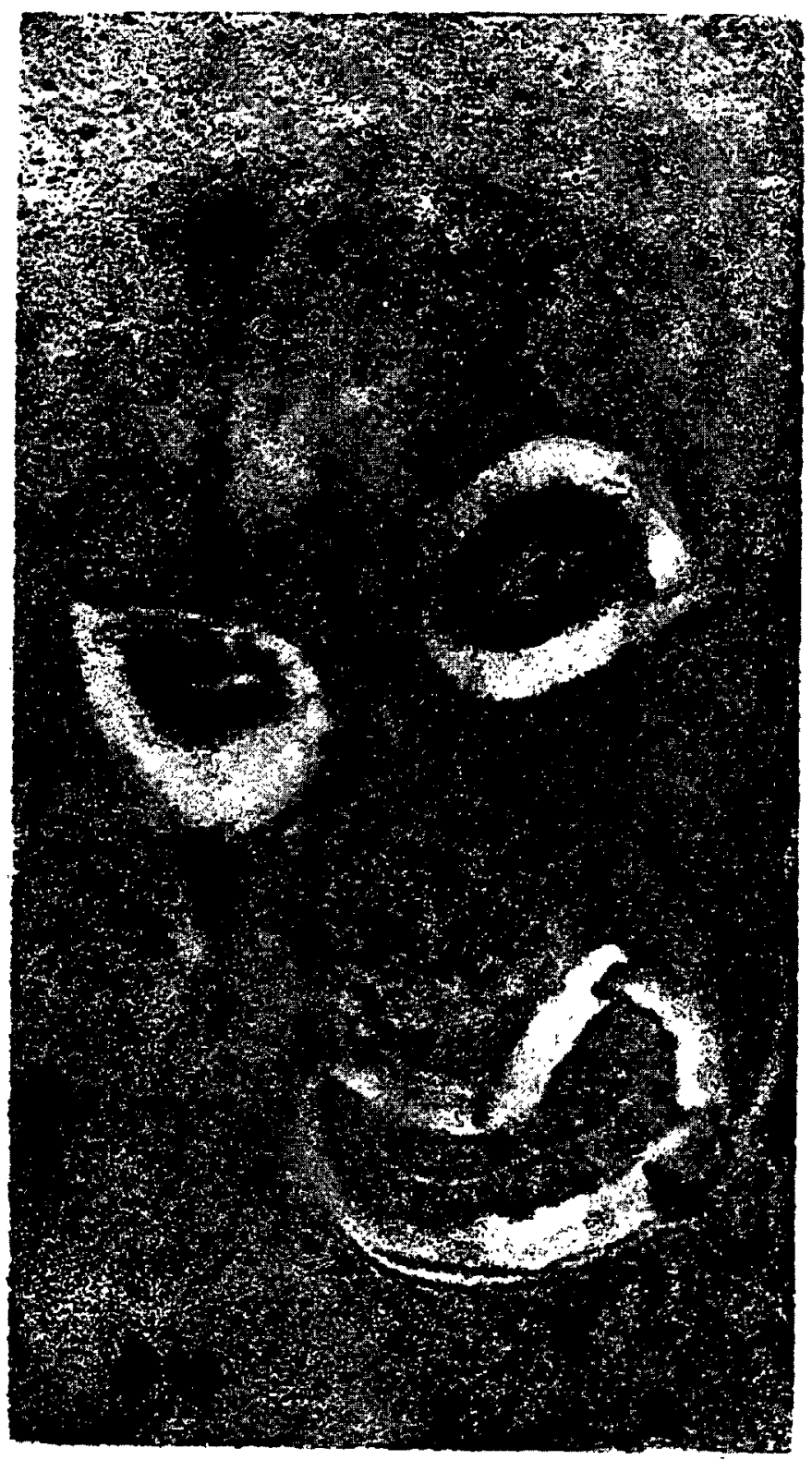

...un certain sentiment d'étrangeté. 
V. \& I. - Vous qui êtes lecteur de Platon, vous tenez des propos antisocratiques.

J. Brault - Oui ... «Connais-toi toi-même", «deviens qui tu es » : ce sont des formules... S'il y a cet effort de connaissance, c'est que ce n'est pas évident. Et non seulement ce n'est pas évident, mais on ne va pas du même au même.

V. \& I. - Il est vrai que dans les dialogues de Platon cette révélation prend la forme d'une surprise.

J. Brault - Exactement. Je décris cela de façon inchoative. Il m'arrive parfois, de me faire reprocher d'être un peu misanthrope. ll est vrai que je m'accorde mieux avec les animaux qu'avec les humains, que je m'accorde mieux avec les plantes qu'avec les animaux... Je n'en suis pas encore aux pierres, mais je pense que ça ne tardera pas. Ce n'est pas la misanthropie qui est en cause, pas du tout, ni la philanthropie, absolument pas. C"est beaucoup plus une question de présence et d'absence un peu emmêlées à soimême. Je me souviens d'une conversation un peu folle avec Robert Marteau: nous nous étions mis d'accord sur le fait que nous n'étions pas vraiment sur terre, mais sans savoir où exactement. Il faisait jouer toute une symbolique dans laquelle je ne voulais pas le suivre, mais je fonctionnais très bien dans ces perspectives, sans aucun effort. C'est peut-être un jeu dangereux: on risque de tomber dans une complaisance à l'absence à soi-même. Une complaisance qui comporte toutes sortes de choses tues, qui se révèlent quand on écrit. Tout cela pour dire que, me relisant et sentant de l'étrangeté, de la distance ... Cela peut s'éprouver sur le plan technique même; par exemple, je n'utiliserais plus certaines techniques d'écriture aujourd hui, mais il n'est pas du tout dit que je ferais mieux, non pas mieux en soi, mais par rapport à ce qu'il y avait à faire, à ce qui était possible alors et à ce qu'il y a à faire maintenant: On connaît des écrivains qui ont retouché leurs textes. et ce n'était pas toujours des bonheurs. Pourquoi aurais-je refait mes poèmes si je n'arrivais pas à les revivre? La question fondamentale est là: je n’y arrive pas. D'autres y arrivent peut-être, pas moi. Je parle des poèmes; les articles. les essais, c'est 'un peu différent, quoique. fondamentalement. c'est sans doute la même chose.

V. \& I. - Dans Chemin faisant, vous avez à la fois repris tels quels et modifié vos essais en les annotant de manchettes.

J. Brault - J'ai dû me battre avec l'éditeur pour obtenir cette typographie. J'ai aussi un peu retouché les textes, par exemple en supprimant des paragraphes qui collaient à une certaine actualité ou des allusions et des références de nature ponctuelle. C'est tout. Il se peut que je fasse un autre recueil d'essais, avèc une autre astuce typographique: une mise en page en mosaïque un peu comme dans les rubriques médiévales, qui pénètrent le texte. Je sais que l'éditeur va protester, mais ce n'est pas très important. Cé sera une façon de revenir sur le texte, plus légèrement que dans Chemin faisant. Si on a écrit sur un écrivain ou une cuvre et qu'on y revient dix ans plus tard, c'est 
évidemment différent; mais ça m'embêterait de refaire le texte, alors je peux ajouter un post-scriptum ou des notes.

V. \& I. - Pourriez-vous ne pas écrire?

\section{J. Brault - Oui.}

V. \& I. - Mais étant donné votre façon d'écrire constamment, cela signifierait une modification complète de votre existence.

J. Brault - Cesser d'écrire dans mes cahiers?

V. \& 1. - Oui. Ma question ne porte pas sur la publication mais sur l'écriture.

J. Brault - Ne plus écrire du tout, ne pas griffonner dans mes cahiers, mes carnets?... Non, je ne peux pas l'imaginer, sauf, bien sûr, pour des raisons, disons, de santé. si je devenais complètement gâteux... De toutes façons. je ne le saurais pas. Non. Je me vois toujours écrivant.

V. \& I. - Autrement dit, l'écriture est liée à votre vie?

J. Brault - Oui, absolument.

V. \& I. - Avez-vous déjà rêvé de ne pas écrire?

J. Brault - ... Oui... Tiens, je l'avais oublié. Dans ce rêve, il ne m'était pas interdit d'écrire, mais je ne le pouvais plus. Du tout. Sans que ça me soit interdit de l'extérieur... Dans ce rêve - il ne faut pas que je le reconstruise. je m'en souviens - je me parlais. Mon astuce (j'étais enfermé sans pouvoir écrire), c'était de me parler. Je n'étais pas en prison, pas nécessairement: c'était une pièce dont je ne pouvais pas sortir. Je n'avais pas le droit de parler à haute voix mais je ruminais. C'est aussi cela, écrire. Et je mémorisais des choses.

V. \& I. - Alors vous êtes tout à fait écrivain...

J. Brault - Ah oui! On ne choisit pas, c'est comme ça! Je suis même allé jusqu'à fantasmer mon dernier livre, il n'a pas de fin: je suis attelé jusqu'à mon dernier soupir! J'ai fait une petite expérience vers 1970. J'ai eu des troubles de l'oreille interne, des difficultés d'équilibre. J'ai dû subir des examens, et on m'a prescrit de garder le lit, allongé sur le dos, sans lecture ni télévision, forcé de méditer. Je ne me suis pas ennuyé un instant. Ça a duré quatre jours; ça n'est rien, quatre jours, mais dans les circonstances, c'est long. Je devais rester à plat, sans oreiller, à regarder le plafond sans faire de mouvement brusque. Je somnolais un peu, forcément. et je m'éveillais la nuit parce que j’avais somnolé le jour. Je me suis laissé aller à la méditation. comme les chats. J'aurais fait un bon chat...

V. \& I. - Vous avez dit qu'écrire, c'est s'adresser à quelqu'un. À qui? 
J. Brault - $\grave{A}$ un inconnu, vaguement imaginé.

V. \& I. - À quelle fin, alors, écrit-on à quelqu'un d'inconnu et d'indéterminé?

J. Brault - C'est le visage évanescent de l'autre qui m'intéresse. Je suis incapable de pratiquer le langage qui s'avale puis s'engendre lui-même. À mes yeux, le langage littéraire est sous tension: il comporte un pôle d'intransitivité et un pôle de transitivité qui est ce quelqu'un, cette présence diffuse. C'est très important cette présence indéterminée qui me soutient, m'aide à donner un sens, une direction à ce que j'écris. J'aime m'adresser à quelqu'un. J'ai toujours aimé le genre épistolaire. Maintenant, hélas! il disparaît. mais pour moi, c'est le genre par excellence.

V. \& 1. - On pourrait dire que vous vous êtes installé à la campagne pour avoir l'occasion d'écrire des lettres...

J. Brault - Oui, et d'en recevoir, ce que j'adore. Autrement tout se fait par téléphone et devient purement fonctionnel. Je considère, dans la pratique de l'écriture, que le langage est référentiel et transitif. Je n'emploierais pas pour autant le mot de "communication", beaucoup trop mobilisé du côté de la sociologie; je ne cherche pas à communiquer ou, comme disent les gens, à "délivrer mon message", qui n'est pas emprisonné. Je n’ai rien de particulier à dire aux gens.

V. \& I. - Vous considérez-vous comme un écrivain québécois?

J. Brault - J'ai déjà écrit qu'il ne faut pas chercher à être écrivain québécois. On l'a beaucoup trop cherché. Il faut écrire. On est québécois, on est au Québec, bon, voilà. Mais écrivain de langue française, oui, ça fait plus de sens. La langue française se pratique avec toutes sortes d'accents. Se vouloir écrivain québécois selon quelque idéologie $X-Y-Z$ ? non. Ce qui ne veut pas dire que je renie certains propos que j’ai tenus dans les années soixante: ils étaient en situation. L'expression «écrivain québécois" peut porter à rire ou à pleurer: elle n'est ni meilleure ni pire que celles d'“écrivain français" ou d'«écrivain italien»: c'est une appellation d'origine.

V. \& I. - Pensez-vous qu'il existe quelque chose qui s'appelle littérature québécoise?

J. Brault - Sur un plan sociologique et historique, oui, j'imagine. C'est un ensemble d'œuvres, de textes, qui ont quelques traits communs, qui se reconnaissent à cette appellation d'origine.

\section{V. \& I. - Y trouvez-vous vos références?}

J. Brault - En partie. C'est comme si on parlait de la littérature écrite en langue allemande par des Autrichiens. qui ont leur histoire, et peut-être aussi quelques différences culturelles. Bon. d'accord. Maintenant, est-ce que Ste- 
fan Zweig est pour toujours et à jamais ứn écrivain̆ de la littérature autrịchienne? Ou Musil? Est-ce qu'on peut les réduire à cela? Leur œuvre est-elle suspendue à cette appellation globale? Non. Je pense que c'est commode, que c'est pratique, que c'est indicatif. Je le dis sans aucun mépris, bien évidemment. Cela forme un ensemble distinct d'autres ensembles, et, finalement. comme toujours, chaque auvre se singularise, est une exception.

V. \& 1. - L'expression "littérature de langue française" serait-elle plus décisive?

J. Brault - Ce qui est vraiment ma patrie d'écrivain. c'est la langue. La langue française, avec cette pratique qui, chez moi, est à la fois locale et pas du tout locale, qui vient de toutes sortes de sources que j'ai arrangées tant bien que mal à ma façon. Je ne nie pas les déterminismes sociaux, culturels, et autres: il est certain que j'ai dans ce que j'écris les réactions de quelqu'un qui est né au Québec. qui y a été éduqué, qui y vit. Certainement. Mais ce n'est pas un programme, ce n'est pas un horizon et ce n'est pas une référence déterminante ou préétablie. Pas du tout.

V. \& I. - Qu'est-ce qu'être écrivain?

J. Brault - Je vais partir d'une anecdote. Il m'est árrivé, je ne sais plus où. d'indiquer que je me considérais comme un écrivain amateur. Chose qui a fait sourire certaines personnes, d'un sourire assez sceptique, et qui a été relevée aussi, je crois, dans une revue, comme de la «fausse modestie». Ca m'a étonné et amusé à la fois de voir ces réactions. Ca m’a étonné parce qu'on a pris la chose comme si je me diminuais ou si je minimisais mes capacités ou mon travail. Alors que j’avais employé l'expression comme on parle d'amateur de musique, de mélomane, en me disant en somme que l'amateur, c'est quelqu'un qui aime faire ce qu'il fait. C'est pour ça que je suis écrivain; pour le plaisir.

V. \& I. - On a reçu cette déclaration comme de la "fausse modestie», à la limite comme une pose étant donné qu'il y a tous ces livres derrière vous. Cette réaction s'inscrit dans un contexte de revendication d'un statut professionnel pour l'écrivain, qui implique une certaine idée de la littérature, un certain rappọrt à l'écriture et au public. Manifestement, revendiquer un statut d'amateur, compte tenu de toute l'épaisseur de sens que vous lui donnez, suppose un tout autre rapport.

J. Brault - Oui, en particulier pour ce qui concerne l'institution littéraire ou culturelle.

V. \& I. - Est-ce que ça signifie que vous vous tenez volontairement à l'écart de cette institution?

J. Brault - Il ne m'est jamais venu à l'idée, même beaucoup plus jeune, de faire carrière d'écrivain, ni même carrière de professeur. Je n'arrive pas à midentifier en profondeur, intérieurement, à un rôle social. Je sais bien 
qu'on n'échappe pas aux rôles, aux étiquettes, aux uniformes symboliques. Je ne voulais pas "arriver" comme écrivain. Ce n'est pas que je m'y refusais, je n'y pensais même pas. Je peux le voir avec du recul maintenant. Alors, j'ai fait des choses comme ça, comme beaucoup; c'est très banal. J'ai commencé doucement, j'ai écrit des choses. j'ai publié dans des revues, sans plan préétabli. Et sans ambition réelle. Je ne voulais pas passer à une revue plus importante, établir des contacts... Je n'ai jamais cherché à devenir ou àttendu d'être salué comme un écrivain, d'en avoir le titre et la reconnaissance que ce titre implique. D'où une certaine liberté intérieure, tout au moins, je le pense. Il n'était pas question de gagner ma vie avec ça, sauf si j’avais été rédacteur, si j’avais écrit de façon, je ne dirais pas alimentaire, mais, justement, professionnelle, comme journaliste, ou autrement.

V. \& 1. - Est-ce écrire dans le même sens, à ce moment-là?

J. Brault - Ce sont des travaux d'écriture, disons. Peut-être que si j'avais vécu dans un autre milieu j’aurais réagi différemment, quoique j'en doute. Comme je l'ai dit tout à l'heure, ça m'a permis de ne pas viser une place dans l'institution. Je ne crois pas. même inconsciemment, entretenir des amitiés littéraires. J'ai des amitiés chez des gens qui écrivent mais ce sont vraiment les personnes qui sont en cause; peut-être les circonstances, que je vais appeler "littéraires", ont-elles favorisé ces rencontres et l'entretien de ces amitiés. Je ne me suis jamais identifié à un groupe littéraire et je n'ai jamais été solidaire d'un mouvement littéraire. S'il y a eu des intérêts communs, des partages de point de vue ou même de valeurs, tant mieux. C'est aussi en ce sens-là que je dis que j’ai été et que je reste un écrivain amateur. 11 m'est arrivé des choses qui, vues de loin ou d'un point de vue social, me confirment dans le rôle d'écrivain, c'est vrai, qu'il s'agisse de prix ou de participations à des rencontres d’écrivains. Mais moi, honnêtement, j’ai toujours fait ça librement, par intérêt, par plaisir, par passion. Je n'ai pas fait carrière, je ne ferai pas carrière. Je ne vise aucune reconnaissance de ce point de vue-là, ce qui n'implique pas que je n'apprécie pas d'être lu.

V. \& I. - Il faut distinguer reconnaissance et réponse.

J. Brault - Justement. Tout amateur que je me reconnaisse, si je prends la peine d'essayer parfois de publier ou de répondre à des offres de publication, c'est parce que, pour moi, écrire, essentiellement, c'est écrire à quelqu'un. Même si l'écriture littéraire en soi est intransitive, on la fait quand même se transiter par des moyens de communication ou tout au moins des possibilités de communication. Je n'ai jamais cherché à avoir un public ou à savoir qui me lisait, quand ça pouvait arriver, mais j'étais heureux, si à un moment donné, quelqu'un lisait mes choses puisque c'est fait pour ça, quand c'est publié. Sinon, on les communique par d'autres moyens beaucoup moins coûteux, moins compliqués. Mais je me considère toujours libre. Par exemple, je ne me suis jamais demandé. en écrivant, ce qu'on allait en penser. Non. Ça ne me vient pas à l'esprit. Pas plus que de chercher à changer de manière. Absolument pas. Pas plus que de me sentir obligé de publier quel- 
que chose parce que ça fait cing ans que je garde le silence. La carrière d'écrivain, c'est un tout petit peu comme le "show bizz". Dans le domaine du spectacle, si on disparait pendant trois ans, il faut vraiment se refaire un public, puis aussi refaire son répertoire parce que tout le monde a entendu tout ce qu'on avait à dire ou à chanter. Il y a un peu de ça chez un écrivain de profession. Et je dirais même que ce n'est pas pour rien, dans le contexte français, que les écrivains se croient obligés ou sont obligés d'habiter à Paris ou dans la région parisienne.

V. \& 1. - Ici, au Québec, on peut dire que les écrivains sont de plus en plus "contraints" d'habiter la région de Montréal.

J. Brault - C'est vrai, même pour les gens de la ville de Québec. Ils le disent. sauf quelques résistants. C'est un exemple, justement, de ce que ça implique de faire du travail d'écrivain reconnu socialement. C'est toujours le même modèle, pratiquement, sauf, peut-être aux États-Unis, mais même là, il y a trois ou quatre grands centres d'attraction. Ce n'est pas le centralisme unique de Paris mais il y a New York. San Francisco. Chicago. Boston. etc. Mais, finalement, c'est toujours la même loi, le même modèle. Dans les contextes étrangers qu'on vient d'évoquer, il arrive que quelqu'un gagne bien sa vie par un travail d'écrivain qu'il considère comme une profession. Ce qui ne veut pas dire qu'il ne se garde pas libre à travers toutes les difficultés que ça comporte. Ce n'est pas ça que je veux dire en revendiquant le statut d'amateur et ce n'est même pas un statut. Puis, je ne le revendique pas vraiment.

\section{V. \& I. - C'est une qualité, ce n'est pas un statut.}

J. Brault - Peut-être, mais ça a été perçu négativement, comme si je me diminuais par fausse modestie, parce qu'il semblerait qu'un écrivain doit nécessairement avoir un statut social patent.

V. \& I. - Vous avez dit tout à l'heure qu'on n'échappe pas aux rôles sociaux ni même aux uniformes symboliques. Mais n'est-ce pas à la condition de nous placer du point de vue qui nous considère comme un être essentiellement social? Est-on vraiment obligé d'accepter cette façon de voir?

J. Brault - Non, mais je crois que ça fait partie du tableau, même personnel. D'abord, dans nos relations avec les autres, ça sert de médiation, toujours, que ça nous fasse plaisir ou non. qu'on le veuille ou pas. Et même dans nos relations avec nous-mêmes, ça occupe une partie du champ. Tout dépend de la façon dont on réagit et dont on valorise cet aspect de la vision de soi-même par soi-même. Autrement dit, si j'essaye de me considérer moi-même et si je me prends pour un écrivain, ça commence à aller mal, à mes yeux en tout cas. Finalement, je fais un travail et je n'ignore pas, bien sûr, que si j'écris un article, qui va être publié dans une revue, je serai logé à une certaine enseigne. Mais si, parce que c'est une revue qui se prétend d'avant-garde, je commence à me forcer pour faire de l'avant-garde, plus ou moins subconsciemment, là, je joue un rôle social. Je réponds à une commande. Ou alors, je peux faire 
l'inverse, je peux faire exprès pour jouer les subversifs de service; ça existe aussi. Il $\mathrm{y}$ a des lieux sociaux pour la subversion; maintenant tout est aménagé, tout est planifié, tout est régulièrement révisé, je dirais environ tous les cinq ans, parce que les lieux forts de la subversion ou de la révolution ou de la révolte se déplacent constamment. L'avant-garde vieillit. Elle est toujours menacée, en ralentissant le pas, de se retrouver à l'arrière-garde. Alors ou bien il faut se refaire de nouvelles jambes, ou bien il faut s'accrocher aux plus jeunes qui sont plus frais, plus vigoureux.

V. \& I. - La «subversion» est subventionnée, ici, de toute façon.

J. Brault - La littérature, ici, est sursubventionnée. On nous fait jouer, parfois, des rôles dans les jurys. On peut le faire dans un état d'esprit, où, finalement, ça sert la carrière: c'est une façon non seulement de se faire reconnaître, mais de se reconnaître soi-même. Mais on peut aussi voir ça comme un service à rendre, un peu comme dans notre métier de professeur. Il y a toutes sortes d'à-côtés qui font qu'on va être sollicité pour ceci ou pour cela et, bon, on peut le faire pour améliorer son dossier - ce que je trouve parfaitement ridicule à tous les points de vue. En tout cas, ça se fait. Ou alors, on peut le faire tout simplement parce qu'on considère que ça fait partie de sa tâche.

V. \& I. - Jusqu'ici nous avons examiné cette question du point de vue de l'institution. Mais une autre aspiration peut définir l'écrivain, celle de faire œuvre. Y a-t-il en vous la volonté de faire une œuvre littéraire?

J. Brault - Non. Ce à quoi j’aspirais adolescent. c'était à autre chose. Il faut revenir à l'adolescence parce qu'il y a là des vérités qu'on occulte en vieillissant; je ne dis pas que ce sont des vérités immuables et éternelles, ni prédéterminantes, mais elles peuvent être intéressantes à regarder rétrospectivement. Moi, tout bêtement, étant donné la situation où je me trouvais. j'aspirais à écrire, à faire de belles choses. Je ne savais pas ce que je voulais dire. (Je ne le sais pas plus dailleurs maintenant). Je voulais que ça soit beau, c'est tout: comme ça, comme on bricole des choses, pour soi-même. Non, il ne m'est jamais venu à l'idée de bâtir une œuvre. J'ai fonctionné un peu au petit bonheur, par des choses ici et là.

V. \& I. - L'idée d'amateur comporte aussi l'idée d'apprenti.

J. Brault - Oui.

V. \& I. - Dans le discours que vous aviez prononcé lors de la remise du Prix Duvernay, vous vous définissiez comme apprenti.

J. Brault - Oui.

V. \& I. - L'idée d'apprenti implique non seulement d'aimer ce qu'on fait mais aussi d'apprendre et de progresser. 
J. Brault - Oui, c'est juste. Mais on continue d'aimer ce qu'on fait malgré certaines répétitions, parce qu'on reste en état de marche, de découverte ou tout au moins de recherche, donc d'apprentissage. J'aime cette notion d'apprentissage que j'ai trouvée chez Cézanne, mais je la portais à l'état latent en moi sans le savoir. Dieu sait si Cézanne n'était pas un poseur, non seulement comme peintre mais aussi comme homme qui a "tenu" jusqu'à la fin de sa vie. Il continuait, comme il disait, à faire ses études en allant sur le motif, deux ans avant sa mort. Et ce n'était pas simplement par modestie. En lui demeurait une jeunesse inaltérable. Un apprenti, en général, est jeune, puis quand il vieillit et qu'il a pris du métier, justement, il devient un professionnel.

V. \& I. - Un maître?

J. Brault - Après. Il va devenir un maître quand il va être vraiment consacré.

\section{V. \& I. - Homme de métier?}

J. Brault - C'est autre chose. I'en ai connu quelques-uns dans ma vie, pas beaucoup. Ca n'existe presque plus maintenant parce que les beaux métiers ont pratiquement été supprimés. L' homme de métier va combiner la maîtrise et ce que j’appellerais l'amateurisme. C'est ce qui m'a frappé en tous cas chez ces quelques hommes de métier que j’ai connus et qui, nécessairement. étaient vieux parce que c'était la dernière génération. Ça m’a frappé à tel point que j'y pense régulièrement et je dirais que les idéaux naîfs que j’évoquais tout à l'heure se sont déplacés plutôt de ce côté-là. Alors il y a donc l'amateur, l'apprenti et l'homme de métier. Je ne dis pas que ça se confond, non, mais c'est en relation étroite. Si jamais on reste en état d'apprentissage constamment, on est en état de recherche, on est en état d'étonnement et je dirais même d'admiration au sens philosophique. Vraiment. Avec peut-être quelques erreurs de perspective qui peuvent en découler mais aussi une certaine fraîcheur de perception. On reste amateur de ce point de vue-là puisqu'on travaille pour lamour du beau métier. La maîtrise qu'on veut atteindre, vers laquelle on tend, est toujours ajournée, parce que, finalement. ce n'est pas l'objet qui importe, c'est la démarche. C'est ce passage en avant (en avant parce qu'on se donne un horizon) qui donne sens à toute l'entreprise. Bien sûr, ça peut être jalonné d'objets qui, dans la circonstance, vont s'appeler des textes mais ce n'est pas vraiment une fin en soi. En ce sens-là, je me dis écrivain amateur. J'ai peut-être tort. Mais c'est ainsi.

V. \& I. - Vous citiez l'exemple de Cézanne. Il permet de très bien saisir l'opposition entre l'artiste amateur et le professionnel. On peut juxtaposer toute l'activité de Cézanne lui-même à l'image qu'en a présenté Zola dans l'Oeuvre. On sait comment Cézanne a réagi; il était un ami d'enfance de Zola, un grand ami. mais Zola n'avait rien compris.

J. Brault - Absolument rien. Zola attachait tout son prix à la profession. C"était un écrivain professionnel et qui se comportait comme tel d'ailleurs. 
surveillant ses tirages et ses comptes. La réussite, pour lui, était extrêmement importante. Et à ses yeux, Cézanne était un raté.

V. \& I. - Oui. Son personnage, Claude Lantier dans l'Oeuvre, peint en fonction du Salon.

J. Brault - Ah oui.

V. \& I. - Et il prépare un "chef-d'œuvre» pour le Salon.

J. Brault - Voilà, c'est ça. Il prépare un «chef-d'œuvre" qui va emporter l'adhésion. C'est exactement l'attitude de qui vise la réussite, qui est ellemême définie par une institution, un ensemble de thèmes sociaux.

V. \& I. - Vous disiez tout à l'heure que. vous n'avez jamais espéré une reconnaissance du statut d'écrivain. Mais il faudrait distinguer cette reconnaissance d'une réponse. Autrement, comment justifier qu'on publie?

\section{J. Brault - D’accord.}

V. \& I. - Qu'est-ce que c'est une réponse?

J. Brault - La réponse au texte, c'est la lecture, chose difficile à apprécier car on n'est pas là, on n'est pas caché dans le coin quand se produit une lecture. On n’a pas cette réponse dans l'instant, comme au théâtre. En littérature, la réponse dont on peut prendre connaissance, c'est habituellement ce qu'on appelle la critique, la critique de compte rendu qui est très variable, et qui elle-même (ce n'est pas un tort que je lui impute) est située dans l'institution littéraire. C"est inévitable parce qu'il y a tellement de nécessités auxquelles elle doit satisfaire. De ce point de vue, il faut se garder une certaine liberté. Je ne méprise pas la critique de compte rendu. On me demande qu'est-ce que tu penses de tel article critique, qu’il porte sur moi ou sur d'autres; ça dépend d'où il vient. Je suis obligé de me référer à son origine. Mais plus profondément, ça dépend de son propos, de l'économie interne de son propos. Je dirais plutôt ça maintenant. C'est plus juste et plus précis. Peu importe l'individu qui signe, c'est l'économie interne du propos qui est déterminante. Il y a des critiques extrêmement intéressantes de ce point de vue-là. Parfois, aussi, on a des échos, très rares, réfractés, de lecteurs, de lectures. Mais je pense que fondamentalement, l'écrivain, comme je le vois. va attendre une réponse et, en même temps, ne pas l'attendre. Il entend une vague rumeur, mais il continue à marcher; il ne va pas s'arrêter sur place - en tout cas, moi je ne m'arrête pas. Et même si la rumeur est tellement faible qu'elle semble pratiquement inexistante, il continue. Écrire. c'est un exercice solitaire.

V. \& I. - Il est de mode maintenant de dire que l'institution littéraire au Québec est surdéveloppée. Mais, est-ce si sûr? Par exemple, il n’y a pas tant de lieux de réponse critique, disons, pour un recueil de poèmes. Où peut-on en trouver un compte rendu? 
J. Brault - Il y a quatre ou cinq lieux qui nous viennent à l'esprit. Dans les journaux, peu, finalement : dans La Presse. Le Devoir...

V. \& I. - À La Presse, on ne parle plus de poésie depuis des années...

J. Brault - Du côté des journaux, à strictement parler, Le Devoir va s'imposer. Le reste, ça va être des revues. des publications spécialisées. Un écho radiophonique peut-être, mais ce n'est pas garanti. C'est tout. II est vrai que notre institution littéraire, par rapport à nos ressources, paraît lourde ou trop présente. Mais finalement, elle est peut-être moins contraignante que d'autres dans d'autres milieux. La tentation de professionnalisme, pour y revenir, n'est pas très forte ici. Il faut quand même être un petit peu absent à soi-même et aux réalités extérieures pour vouloir faire profession d'écrivain. au sens habituel du terme, au Québec. Vouloir gagner sa vie comme écrivain et non pas comme scripteur ou comme rédacteur. Alors, on a le choix entre se conter des hiștoires, se bercer d'illusions, être très amer et même vinaigré, ou se dire: «bien, je prends appui sur l'obstacle et je travaille librement; je gagnerai ma vie autrement ". Il y en a qui aimeraient écrire toute la journée; moi pas. La situation me convient parfaitement. Et puis, finalement, je me dis que c'est assez léger: si je ne collabore pas à telle revue pendant deux ans, ça ne compromet rien, ça ne me fait rien perdre, absolument pas, je n'ai pas de terrain à regagner et ainsi de suite. Je trouve que je me suis accommodé de ces conditions assez facilement. Je n'ai eu aucun mérite et il n’y a donc ni modestie ni "fausse modestie» de ce point de vue.

V. \& I. - Si nous considérions l'acte même d'écrire, de façon extrêmement élémentaire. très concrète. Vous écrivez un poème. Comment cela se passe-t-il?

J. Brault - C'est imprévisible, jusqu'à un certain point. D'habitude, je travaille au petit bonheur dans mes cahiers, ou sur des feuilles volantes. Je griffonne. Je rédige. Quelque chose peut cristalliser. Ce qui importe c'est ça, ce que j'appelle la cristallisation ou la matrice fécondante. Il y a quelque chose qui se passe. Est-ce que c'est une perception plus aiguë? Je ne le sais pas. Ce sont des mots. c'est du langage qui, tout à coup. devient attractif, qui tiraille d'autres langages. C'est ça. le poème; parce que le poème n'est pas fait d'heureux passages, de belles expressions, ici et là, comme on disait autrefois de beaux vers. Le poème est une espèce de noyau, finalement. Ensuite. je procède par découpage. montage, collage et ainsi de suite. Puis je laisse dormir. Quand un texte est plus long (parce que la longueur, à mes yeux. fait partie de la forme) je travaille de façon plus artisanale, avec crayons de couleur, papier quadrillé; je me fais même des courbes. des graphiques. Ça ne s'oppose pas à ce qu'on appelle l'inspiration avec un I majuscule. Il s'agit de dégager la forme sans la fracasser, un peu comme certains archéologues dégagent une poterie fragile. Quand on en arrive à la révision et à la correction, on se demande si ça va aller ou pas et alors toutes sortes de choses peuvent survenir. J'avais jeté une feuille au panier. Puis, cette feuille qui était froissée a roulé dans un coin où elle est restée trois jours. Tout à coup, je l'ai 
retrouvée. Je l'ai défroissée puis je me suis dit que ça irait bien dans un bouquin. Il s'agissait de l'En dessous l'admirable; c'est devenu (parce que l'En dessous l'admirable n'est pas un recueil, c'est un petit livre, un seul poème) un moment du poème presque tel quel, à mon étonnement.

V. \& I. - Il n'y a donc pas un moment particulier de l'écriture. Vous écrivez tout le temps dans des cahiers, des carnets, sur des bouts de papier, puis, intervient l'opération de découpage, collage, correction. N'y a-t-il pas là une technique pour repousser l'écriture elle-même, qui est angoissante?

J. Brault - Je reste persuadé que ce que j'appelle le travail artisanal (jouer avec toutes sortes de choses, être concret, jouer des crayons de couleur...) est une façon de me désangoisser. Certainement. De me désangoisser à toutes sortes de points de vue. 11 y a l'angoisse superficielle, qui tient d'une anxiété immédiate, mais aussi l'angoisse plus profonde. Et. c'est là qu'écrire prend toutes sortes de sens, parce que je reste persuadé qu'on écrit pour des motivations qui nous échappent en dernière analyse. Certains ont prétendu que c'est parce qu'on vit mal, parce que la vie ne nous satisfait pas. D'autres, au contraire, vont dire que c'est de la surabondance. Bon. On a présenté toutes sortes de simili-théories là-dessus mais il n'en reste pas moins qu'il y a des fantasmes, je dirais même qu'il y a une pulsion à l'oeuvre, certainement, qui peut être plus variable qu'on le croit. Oui, il s'agit d'une pulsion. Une pulsion de mort. Ce n'est pas du tout négatif. Pulsion de mort, parce que vivre c'est se sentir mourir. Vivre en ayant conscience de vivre, c'est être mourant. Les gens s'imaginent qu'on est morbide. qu'on fait de l'humour noir, mais pas du tout. La courbe de la vie est ainsi faite. C'est au moins une des choses que la nature nous enseigne. Il y a donc cette pulsion de mort qui est à l'œuvre. Alors, pourquoi se donner ce travail, ce grand détour de l'écriture? Parce que, pour moi, c'est un détour. C'est pour ça qu'on parle de l’écriture oblique. On écrit plutôt que d'y aller en ligne droite ce qui, souvent, consisterait à se taire ou a recourir au langage comme à un instrument, à se servir du langage. Alors, pourquoi écrire, sinon pour temporiser, aménager le temps.

V. \& I. - Est-ce qu'on peut dire tempérer?

J. Brault - Bien, on peut dire tempérer, on peut dire temporiser, on peut dire temporaliser. Tempérer cette accélération qui consiste à vivre, parce que vivre, ce n'est pas simplement passer, c'est passer en allant de plus en plus vite. C'est ça vieillir. La sensation de vieillir, elle est là. Donc, tempérer, de ce point de vue, réaménager aussi. Je vais me permettre un grand mot, mais ce n'est pas uniquement un grand mot, c'est aussi une grande chose: il s'agit de réaménager une transcendance dans l'immanence à soi-même. Donc, c'est se mettre à croire, il faut bien le dire. À croire. On ne peut pas écrire si on n'est pas croyant. Si on ne croit pas à quelque chose ou en quelque chose. Certainement. Écrire, c'est se mettre à croire et non pas simplement à savoir, ou à agir. Il faut le reconnaître avec peut-être toutes les aberrations que ça peut comporter sur le plạn idéologique quand on se met à penser à ce sujet ou à en faire la théorie. 
V. \& I. - Sans tomber dans ces aberrations, ni théoriser, vous avez parlé de transcendance; vous avez dit "croire". Ce sont des mots chargés de tellement de sens...

J. Brault - Évidemment, ils peuvent comporter diverses significations donc, référer à diverses réalités. D’abord, si on parie de transcendance par rapport au flux temporel, il est certain que c'est une façon, je ne dirais pas simplement de magnifier, mais d'éterniser l'instant qui reste fugitif, par expérience plutôt que par définition. De l'éterniser non pas à l'instant où on écrit: le texte écrit va devenir un correcteur d'instantanéité. Et je pense que certaines expériences de la lecture peuvent répondre effectivement de cette éternisation de l'instant. Pas simplement par les thèmes mais parce que l'on vit même en lisant et en étant, en quelque sorte, comblé temporellement par la lecture. C'est une façon d'être hors du flux temporel ou tout au moins à côté. je n'oserai pas dire au-dessus. Il y a ça. Ça semble aberrant du point de vue d'une certaine logique qui n'est que bi-dimensionnelle. C'est dans ce sens-là que j'ai parlé de croyance parce qu'on ne peut pas. je vais me limiter à cela pour l'instant, on ne peut pas établir ça rationnellement. On ne peut pas atteindre à une démonstration qui va combler l'esprit analytique et géométrique ou démonstratif. On ne peut même pas en faire une démonstration à d'autres points de vue, c'est une chose à laquelle on adhère. C'est une valeur qu'on sécrète et qu'on projette, appelons ça un espoir, une illusion qui aide à vivre. On adhère de telle façon que n'étant pas capable d'en répondre à la satisfaction de l'esprit critique, j'ai bien employé le mot de «croyance». C'est quelque chose en quoi je crois. Un petit peu comme je vais croire à l'amour de quelqu'un. Je ne peux pas le démontrer. D'ailleurs, si je commence à le démontrer, c'est mauvais signe, qu'il s'agisse de l'amour de quelqu'un ou de mes propres sentiments. Je pense que lorsqu'on arrive dans l'ordre des valeurs esthétiques, on arrive à un certain nombre de croyances qui ne sont pas uniquement irrationnelles. On peut faire beaucoup de chemin dans l'ordre analytique, mais en dernier recours l'horizon fuyant vers lequel on se dirige, on est obligé d'admettre qu'il appelle une adhésion. Écrire, de ce point de vue-là, c'est faire confiance. 